BMJ Open Sport \& Exercise Medicine

\section{Bubble concept for sporting tournaments during the COVID-19 pandemic: Football Club World Cup}

To cite: Massey A, Lindsay S, Seow D, et al. Bubble concept for sporting tournaments Football Club World Cup. BMJ Open Sport \& Exercise Medicine 2021;7:e01126. doi:10.1136/

Accepted 8 June 2021 during the COVID-19 pandemic: bmjsem-2021-001126

\section{ABSTRACT}

Objectives To report the person-to-staff transmission of COVID-19 with the implementation of a bubble concept that included testing, hygiene, distancing and monitoring strategies to mitigate risks.

Methods A prospective case series included all staff on-site involved in the Football Club World Cup. The tournament was conducted within 'bubbles'. All personnel travelling to the tournament were required to be in possession of a negative RT-PCR test within 72 hours of arrival and subjected to a testing schedule during the tournament. Each location was assigned a COVID-19 protocol enforcement officer to ensure adherence to regular testing, hygiene measures, physical distancing and daily symptom reporting (via ScribePro app).

Results The study involved 70 recruited staff with a combined 1321 test days on the symptom checker app. The mean number of days completed on the symptom checker app was 18.87 days (range: 7-28). Of the five questions asked as part of the daily symptom checker, only one was answered positively $(0.015 \%)$. This individual was isolated, assessed within 20 min and tested. The initial diagnosis was likely a non-COVID-19-related viral illness. Further testing returned three negative tests during the remainder of the tournament.

Conclusions There was no person-to-staff transmission of COVID-19 during the tournament within our sample. The organisation of a sporting tournament during the COVID-19 pandemic is possible with risk mitigation strategies. These strategies include setting up a bubble with regular testing, hygiene measures, physical distancing and daily symptom reporting.

\section{(C) Author(s) (or their} employer(s)) 2021. Re-use permitted under CC BY-NC. No commercial re-use. See rights and permissions. Published by BMJ.

${ }^{1}$ Federation Internationale de Football Association, Zurich, Switzerland

${ }^{2}$ Liverpool Football Club, Liverpool, UK

${ }^{3}$ National University Health System, Singapore

${ }^{4}$ ScribePro, Glasgow, UK

Correspondence to Dr Andrew Massey; andrew.massey@fifa.org

\section{INTRODUCTION}

Football is a sport played and watched by billions of people across the world. ${ }^{1}$ The sport has wide-reaching social, health and economic implications on all aspects of society. ${ }^{2}$ As world football's governing body, the FIFA has the responsibility and mandate to provide appropriate guidance and recommendations to member associations and their stakeholders to mitigate the consequences of disruptions caused by the COVID-19 pandemic.

\section{Key messages}

What is already known

- Staging a sporting tournament in the context of COVID-19 carries a transmission risk.

- Regular testing, hygiene measures, physical distancing and daily symptom reporting can be employed to mitigate the transmission risk within a sporting tournament.

What are the new findings

- Testing, hygiene, distancing and monitoring strategies within a 'bubble concept' can minimise the transmission of COVID-19.

- Symptom-reporting apps can be useful in alerting the individuals and clinicians to the early signs of infection. This can allow swift isolation policies to be introduced and mitigate the transmission risk of COVID-19.

In response to the outbreak of the COVID19 , severe restrictions have been imposed on every facet of life throughout the world. ${ }^{3}$ Football likewise has been affected, with many football-related activities being suspended at some point, in almost every part of the world. ${ }^{45}$ The effects of the pandemic on sport have not been limited to football, and there has been a widespread impact on the staging of sporting events and competitions globally. ${ }^{6}$ Major sporting tournaments have considerable economic, social and physical benefits, which in the context of a global pandemic need to be balanced by any infection risks associated with the sport and any concerns associated with mass gatherings and travel to the event. ${ }^{78}$

FIFA is responsible for 14 competitions (four in association with the IOC) and three e-competitions (table 1). Since the pandemic outbreak, FIFA cancelled or postponed five competitions. Notably, FIFA President Gianni Infantino has stated, 'our first priority, our first principle, the one we will use for our competitions and encourage everyone to 
Table 1 FIFA competitions

\begin{tabular}{llll}
\hline & Men's competition & Women's competition & E-competitions \\
\hline FIFA & World Cup & World Cup & E-World Cup \\
& U-20 World Cup & U-20 World Cup & E-Club World Cup \\
& U-17 World Cup & U-17 World Cup & E-Nations Cup \\
& Club World Cup & & \\
& Beach Soccer World Cup & & \\
& Futsal World Cup & & \\
\hline \multirow{5}{*}{ Staged together with the IOC } & Blue Stars/Youth Cup & & \\
& Men's Olympic Football & $\begin{array}{l}\text { Women's Olympic Football } \\
\text { Tournament }\end{array}$ & Tournament \\
& Men's Futsal Youth Olympic Women's Futsal Youth & \\
& Football Tournament & $\begin{array}{l}\text { Olympic Football } \\
\text { Tournament }\end{array}$ & \\
& & & \\
& & &
\end{tabular}

follow is that health comes first. ${ }^{9}$ The first competition staged by FIFA following the outbreak of the pandemic has been the FIFA Club World Cup (FCWC). This was initially planned for December 2020 in Qatar but then was rescheduled for 1-11 February 2021 and further amended to 4-11 February 2021 on the withdrawal of one team due to quarantine-related measures imposed in their home country.

To the authors' knowledge, there have been no previous data published in the context of COVID-19 transmission within a sporting bubble concept. The purpose of this study was to report the person-to-staff transmission of COVID-19 with the implementation of a bubble concept that included testing, hygiene, distancing and monitoring strategies to mitigate risks. These data can provide valuable information regarding the possibility of holding sporting tournaments within the COVID-19 pandemic.

\section{METHODS}

\section{Study design and eligibility criteria}

A prospective case series included all staff on-site in the host country (Qatar) involved in the FCWC. All staff on-site were eligible for inclusion and have agreed for their anonymised data to be used in this study. The exclusion criteria were staff working remotely. The competition involved five confederations from the following countries: Brazil, Egypt, Germany, Mexico, Qatar and South Korea. This included six teams consisting of players, staff and dignitaries.

\section{'Bubble concept'}

The tournament was conducted within 'bubbles'. Fundamental to this bubble concept was isolating players, staff and dignitaries within tight physical locations to limit the contact with personnel outside of individual bubbles. FIFA medical, safety and operation teams collaborated with the local organising committee of the FCWC to put measures and legislation in place to ensure that the competition was completed with minimal health risks. This was in keeping with FIFA's risk assessment for mass gatherings ${ }^{10}$ and in line with the WHO best practice. ${ }^{11}$ Risk mitigation strategies were implemented in line with FIFA risk assessment and FIFA international match protocol (box 1). ${ }^{12}$

All personnel travelling to the tournament were advised to perform an RT-PCR test 2 weeks prior to the competition. Further, they were required to be in possession of a negative RT-PCR test within 72 hours of arrival in Qatar. Players and staff were isolated until the 'airport' test result was determined to be negative. All personnel were subjected to a testing schedule. This was a 'fluid' testing regime designed to test players and staff every 3-6 days in response to any possible positive tests within the bubble and the wider environment. All tests were performed using a nasopharyngeal and oropharyngeal swab taken by a trained medical professional. Testing was conducted by the Qatar Ministry of Public Health using Thermo Fisher RT-PCR, Roche-Cobas 6800 or Cepheid GeneXpert platforms within established local laboratories. Indeterminate samples were repeated, as necessary.

During the tournament, all areas were strictly policed and regularly sanitised to avoid contamination. All players and staff were alerted of the protocols associated with the bubble concept. Each location was assigned

Box 1 Risk mitigation strategies to decrease the likelihood of COVID-19 transmission

Completion of FIFA risk assessment tool.

- RT-PCR testing programme to include pretravel testing.

- Contact tracing.

- Physical distancing.

Symptom monitoring

- Hygiene measures.

- Minimise on-location staff.

- Educational materials/resources.

- Bubble concept.

- Reduce activities that would increase physical contact (media duties). 


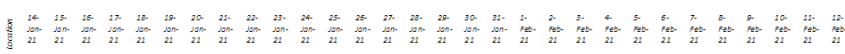

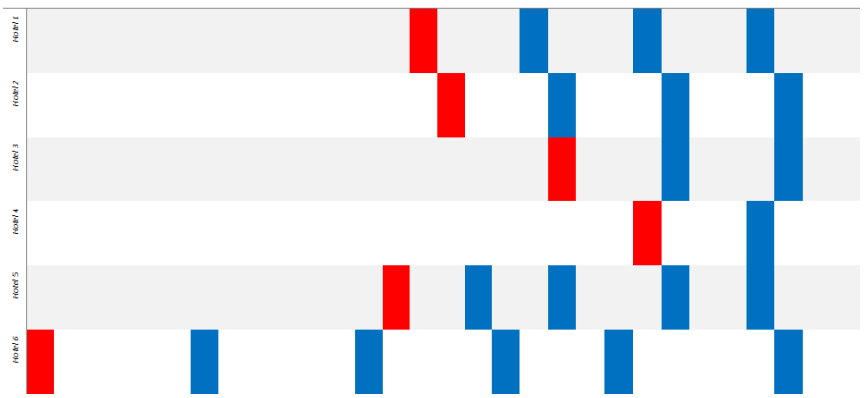

Figure 1 Testing protocol. Blue colour indicates testing at bubble hotel. Red colour indicates testing at airport.

a COVID-19 protocol enforcement officer to ensure adherence to regular testing, hygiene measures, physical distancing and daily symptom reporting. A summary of the individual testing regime and bubbles is illustrated in figure 1.

\section{Daily symptom reporting via ScribePro app}

All staff were required to perform a daily symptom checker generated and notified to them via the ScribePro app (ScribeProTeam, ScribePro, UK; figure 2). ScribePro is an innovative multiuser, multisport medical record system that allows secure, rapid and accurate recording and monitoring of medical injury, illness and prescribing interactions with a highly granular timeline of all data points. ${ }^{13}$ The COVID-19 reporting function is a key enabler of daily symptom reporting harmonised to WHO standards. ${ }^{11}$

Notifications were sent to each staff member at a predetermined time daily, inviting them to complete the daily symptom reporting questions (box 2). The notification was sent via short messages service and email, with only one response required. Responses were updated in real time and reviewed within the medical officer's ScribePro app. Any positive symptom declared resulted in the staff immediately being isolated. A simultaneous real-time change of status within the medical officer's ScribePro app informed of a positive response and this prompted immediate consultation.

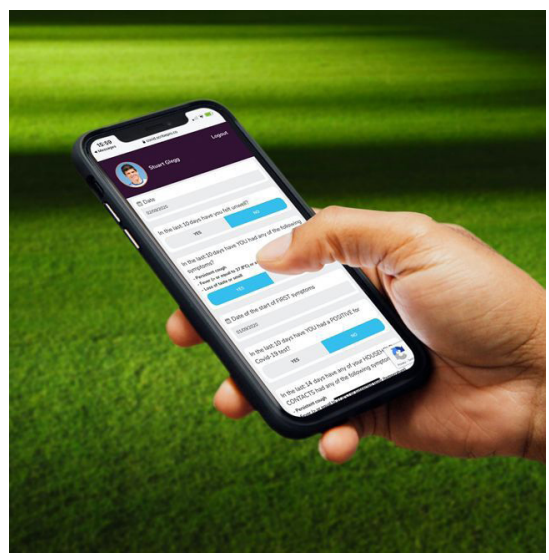

Figure 2 ScribePro app.

\section{Box 2 Questions in the symptom checker}

In the last 10 days, have you felt unwell?

In the last 10 days, have you had any of the following symptoms? Persistent cough.

Fever $\left(\geq 37.8^{\circ} \mathrm{C}\right)$ or suspected fever (temperature). Loss of taste or smell.

- In the last 14 days, have you had a positive test for COVID-19?

- In the last 14 days, have any of your household contacts had any of the following symptoms?

Persistent cough.

Fever $\left(\geq 37.8^{\circ} \mathrm{C}\right)$ or suspected fever (temperature).

Loss of taste or smell.

- In the last 14 days, have you been in contact with a COVID-19 confirmed or suspected case?

\section{Statistical analysis}

All statistical analyses were performed using R V.3.5.1 (R Foundation for Statistical Computing, Vienna, Austria). Descriptive statistics expressed continuous variables as mean \pm SD and categorical variables as frequencies with percentages.

\section{Patient and public involvement}

It was not possible to involve patients or the public in the design, or conduct, or reporting, or dissemination plans of our research

\section{RESULTS}

The study period was between 14 January 2021 and 11 February 2021. Not all personnel travelled to the tournament on the same day. The mean size of a team delegation was 60.16 personnel (range: $41-77$ ). A total of 70 recruited staff were on-site in the host country to help deliver the competition ( 54 males, 16 females). The symptom checker app detailed a combined 1321 test days (screening commenced on the day of travel to the tournament and finished on the tournament's final day). Of the 1321 days, there were $1170(89 \%)$ completed checks by the staff. Only 31 of the 70 staff members $(44.29 \%)$ completed the symptom checker on all the days. The mean number of days completed on the symptom checker app was 18.87 days (range: 7-28).

Of the five questions asked as part of the symptom checker daily, only one was answered positively $(0.015 \%)$. This individual was isolated, assessed within $20 \mathrm{~min}$ and tested. The initial diagnosis was likely a non-COVID19-related viral illness. Further testing returned three negative tests during the remainder of the tournament.

\section{DISCUSSION}

COVID-19 is a novel disease and as the scientific community gathers new information, measures and practices will have to be adapted accordingly. Therefore, the measures and practices described in this study demonstrate an approach based on the current understanding of the transmission of COVID-19 and serve the guiding principle of 'health comes first' to prevent the spread or 
reintroduction of COVID-19. For this tournament, FIFA conducted a risk assessment in collaboration with the local government and public health authorities, consistent with the WHO best practices. This involved strict measures that were implemented to minimise the health risk to players and staff alike. These data have immediate translational benefit that an international football tournament can be staged and conducted safely, with minimal to no transmissibility of COVID-19 when appropriate risk mitigating factors are enforced. The number of personnel within the bubble groups was maintained at a minimum while still providing operational integrity. As our understanding of COVID-19 is constantly evolving, so have and will the defined criteria to screen for those at risk. Therefore, the technology used to screen players and staff must always be updated as new information and guidance are surfaced. Medical record systems such as ScribePro can allow for these changes to be made easily and rapidly.

This is the first study to report on the transmission of COVID-19 among tournament staff members while on-site in a bubble concept in combination with regular testing, hygiene measures, physical distancing and daily symptom reporting (via a symptom checker app). Importantly, the results yielded no person-to-staff transmission. The rate of COVID-19 in this study did not appear to be related to the national rates of COVID-19 in the host country, which saw a weekly rise of $22.33 \%$ in confirmed cases in the first week of the tournament to 14.9 per 100 $000 .{ }^{14}$ This would imply that the bubble concept in association with a risk assessment approach based on scientific evidence supporting regular testing, hygiene measures, physical distancing and daily symptom reporting can be useful and effective. It is known that indoor environments can present a higher risk than outdoor environments. The personnel in this report predominantly conducted their work in an indoor environment.

Despite all subjects having had to undergo international travel to arrive at the host country, the predeparture tests appeared to ensure that those entering the bubbles were already at low risk of transmitting COVID-19. The use of the ScribePro app daily served to remind staff of the importance of adherence with protocol and provided documentation. While limited positives were reported, it enabled medical teams to contain the risk of spread within distinct bubbles. COVID-19 reporting is a key function enabling daily reporting of symptoms and contacts harmonised to WHO standards. ${ }^{11}$

\section{Limitations}

Although comprehensive in testing all members of the bubble, findings are presented with caution, noting the study's limitations. Case numbers were small and limited to staff members, limiting the applicability of findings related to transmission. Future studies should further explore the validity of the 'bubble concept' with COVID-19 reporting tools.

\section{CONCLUSION}

This is the first study to report a bubble concept that included the use of a symptom checker app within the delivery of a sporting tournament. Following the WHO and risk assessment guidance, this tournament was hosted with incidence lower than the general population. There was no person-to-staff transmission during the tournament within our sample. This shows that with regular testing, hygiene measures, physical distancing and daily symptom reporting, the transmission of COVID-19 can be minimised to allow a sporting tournament to be planned and delivered with minimal health risks.

Contributors All authors were involved in the conception, discussions and writing of the manuscript.

Funding The authors have not declared a specific grant for this research from any funding agency in the public, commercial or not-for-profit sectors.

Competing interests JG and DJL report grants from Scottish Enterprise, grants from Innovate UK, during the conduct of the study; other from ScribePro, outside the submitted work.

Patient and public involvement Patients and/or the public were not involved in the design, or conduct, or reporting, or dissemination plans of this research.

Patient consent for publication Not required.

Ethics approval This study involves human participants and was approved by an ethics committee(s) or institutional board(s).

Provenance and peer review Not commissioned; externally peer reviewed.

Data availability statement Data are available upon reasonable request.

Open access This is an open access article distributed in accordance with the Creative Commons Attribution Non Commercial (CC BY-NC 4.0) license, which permits others to distribute, remix, adapt, build upon this work non-commercially, and license their derivative works on different terms, provided the original work is properly cited, appropriate credit is given, any changes made indicated, and the use is non-commercial. See: http://creativecommons.org/licenses/by-nc/4.0/.

\section{ORCID iD}

Dexter Seow http://orcid.org/0000-0003-1769-9244

\section{REFERENCES}

1 Nielsen Sports. World football report. Available: https://www.nielsen. com/uk/en/insights/report/2018/world-football-report/ [Accessed 02 May 2021].

2 Katsarova I, Halleux V. Eu sports policy: going faster, aiming higher, reaching further. European Parliament, 2019.

3 Fauci AS, Lane HC, Redfield RR. Covid-19 - Navigating the Uncharted. N Engl J Med 2020;382:1268-9.

4 Drewes M, Daumann F, Follert F. Exploring the sports economic impact of COVID-19 on professional soccer. Soccer \& Society 2021;22:125-37.

5 Beiderbeck D, Frevel N, von der Gracht HA, et al. The impact of COVID-19 on the European football ecosystem - a Delphi-based scenario analysis. Technol Forecast Soc Change 2021;165:120577.

6 McCloskey B, Zumla A, Ippolito G, et al. Mass gathering events and reducing further global spread of COVID-19: a political and public health dilemma. Lancet 2020;395:1096-9.

7 Parnell D, Widdop P, Bond A. COVID-19, networks and sport. managing sport and leisure, 2020.

8 Ludvigsen JAL, Hayton JW. Toward COVID-19 secure events: considerations for organizing the safe resumption of major sporting events. managing sport and leisure, 2020.

9 The Fédération Internationale de Football Association. Fifa president outlines three immediate priorities for football. Available: https:// www.fifa.com/who-we-are/news/fifa-president-outlines-threeimmediate-priorities-for-football [Accessed 02 May 2021].

10 The Fédération Internationale de Football Association. Fifa, who and football stakeholders draft medical considerations and risk assessment tool. Available: https://www.fifa.com/who-we-are/news/ 
fifa-who-and-football-stakeholders-draft-medical-considerationsand-risk-assessm [Accessed $01 \mathrm{Apr}$ 2021].

11 World Health Organization. A guide to WHO's guidance on COVID-19. Available: https://www.who.int/news-room/featurestories/detail/a-guide-to-who-s-guidance [Accessed 01 Apr 2021].

12 The Fédération Internationale de Football Association. Fifa international match protocol. Available: https://www.fifa.com/who- we-are/news/bureau-of-the-fifa-council-approves-internationalmatch-protocol-and-adapts-rule [Accessed 02 May 2021].

13 ScribePro. Available: www.scribepro.co [Accessed 02 May 2021].

14 World Health Organization. Who COVID-19 case definition.

Available: https://www.who.int/publications/i/item/WHO-2019nCoV-Surveillance_Case_Definition-2020.2 [Accessed 02 May 2021]. 